\title{
Differential influence of hands posture on mental rotation of hands and feet in left and right handers
}

\author{
Silvio Ionta • Olaf Blanke
}

Received: 19 May 2008 / Accepted: 5 March 2009 / Published online: 27 March 2009

(C) Springer-Verlag 2009

\begin{abstract}
The representation of the body in the brain is continuously updated with regard to peripheral factors such as position or movement of body parts. In the present study, we investigated the effects of arm posture on the mental rotation of hands and feet. Sixteen right-handed and ten left-handed participants verbally judged the laterality of visually presented pictures of hands and feet in two different postural conditions. In one condition they placed their right hand on their right knee and their left hand behind the back, in the other condition the hand position was reversed. For right-handed participants response times for the laterality judgment of right hands increased when participants kept their right hand behind the back. This was not found for images of the left hand nor for images of the feet. For the left-handed participants, there was no effect of arm posture on hand or feet stimulus judgments. Thus, the body-part posture effect on mental rotation was found to be specific for the side and the body part for which the posture was modified only in right-handed participants, but it was absent for left-handed participants. For both samples, we also found a progressive disruption of the mental rotation function depending on the view from which the body parts
\end{abstract}

Present Address:

S. Ionta $(\bowtie) \cdot$ O. Blanke

Laboratory of Cognitive Neuroscience,

Brain-Mind Institute, Ecole Polytechnique

Fédérale de Lausanne, Station 19, 1015 Lausanne, Switzerland

e-mail: silvio.ionta@epfl.ch

S. Ionta

ITAB, Institute for Advanced Biomedical Technologies,

G. D'Annunzio University Foundation, Chieti, Italy

O. Blanke

Department of Neurology, University Hospital,

1211 Geneva 14, Switzerland were seen (i.e. dorsal, thumb/big toe, palm/plantar, little finger/toe). Posture and view effects on body parts representations are discussed with respect to proprioception, handedness, visual familiarity and the influence of anatomical joint constraints on motor imagery.

Keywords Mental rotation - Motor imagery · Body representation $\cdot$ Posture $\cdot$ Hand dominance

\section{Introduction}

Mentally simulated and physically executed spatial transformations share similar temporal and kinematic properties (Parsons 1994; Parsons et al. 1995; Decety et al. 1989, 1991; Gerardin et al. 2000; Shepard and Metzler 1971) and the time required to simulate a motor act is proportional to the time needed to actually perform the movement (Parsons et al. 1995; Sirigu et al. 1996). This similarity between real and simulated movements is further associated with proportional neurovegetative activation during physical effort and during the mental simulation of the same effort (Decety et al. 1991) and by specific facilitation of event-related motor potentials during the simulation of motor acts (Fadiga et al. 1995). In agreement with further studies using neuroimaging (Kosslyn et al. 2001), transcranical magnetic stimulation (Ganis et al. 2000) or clinical techniques (Sirigu et al. 1996), it has been argued that mental imagery shares neural mechanisms with movement planning (Decety et al. 1989) and movement execution (Parsons et al. 1995; Gerardin et al. 2000; Grezes and Decety 2001).

A special kind of mental spatial transformation is mental rotation, a cognitive task in which participants are asked to "rotate" mental representations of two-dimensional and three-dimensional objects (Shepard and Metzler 1971). 
When mental rotation concerns a body part, participants tend to imagine the movement of their corresponding body part as though it were moving from its actual position to the target position (Parsons 1994). Mental rotation for body parts engages an anatomically interconnected brain systems implicated in the integration of sensorimotor information (Bonda et al. 1995; Kosslyn et al. 1998; Parsons et al. 1995). The importance of sensorimotor mechanisms in body part mental rotation has been shown by distinct effects due to the stimulus, view, laterality and orientation (Gentilucci et al. 1998; Parsons 1987). Thus, if people are asked to judge the laterality of visually presented pictures of hands and feet, the stimuli's view (i.e. dorsum, thumb/big toe, palm/plantar, little finger/toe) will determine different behavioural responses (Ionta et al. 2007). In the same way, angular disparity between the stimulus orientation and its alignment to the vertical is positively correlated with RTs following a psychophysical profile non-monotonic at $180^{\circ}$ (Shepard and Metzler 1971; Parsons 1994; Parsons et al. 1995; Decety et al. 1989, 1991; Gerardin et al. 2000; Ionta et al. 2007).

Recently, Ionta et al. (2007) showed that hand posture adopted by participants during the mental rotation of hands modifies their behavioural responses in a specific way. When participants were holding both hands behind their back, RTs for hand judgments were increased compared to when the same task was performed with both hands on their knees. This postural effect was absent for pictures of feet, suggesting body-part specific posture effects in mental body representations. The present study extended our previous study by asking participants to make verbal laterality judgments of the same pictures of left and right hands and feet but now with either their right hand behind their back (and the left hand on the left knee) or with the reverse condition (left hand behind the back and right hand on the right knee). We predicted that sidespecific postural effects (i.e. holding the right hand behind the back) would alter the performance for pictures showing right but not left hands and vice versa) and that these effects are absent for pictures of feet. This was tested in right-handed participants (experiment 1) and left-handed participants (experiment 2).

\section{Methods}

\section{Participants}

In experiment 1 , sixteen healthy participants ( 6 female) aged $18-29$ years $(M=21.9$ years, $\mathrm{SD}=2.8)$ were enrolled in the experiment. All participants were right-handed according to a standard handedness inventory (Briggs and Nebes 1975). On this 5-point scale (1 is considered as strongly left-handed and 5 as strongly right-handed) the average handedness score of the group was 4.5. Experiment 2 was carried out by ten left-handed healthy participants ( 3 female) aged $22-34$ years $(M=26.9$ years, $\mathrm{SD}=4)$. The average handedness score of this group was 1.7 . The protocol was approved by the local ethics committee and the research was conducted in accordance with the 1964 Declaration of Helsinki. Written informed consent was obtained prior to the participation.

\section{Materials}

The experimental stimuli consisted of naturalistic pictures of hands or feet, presented one at a time on a computer screen (Fiorio et al. 2006). Left hands and feet were mirror images of the original right ones. Stimuli could be presented in one of four different views (dorsum, little finger/ toe, thumb/big toe, palm/plantar) and oriented in one of six clockwise orientations from the upright $\left(0^{\circ}, 60^{\circ}, 120^{\circ}\right.$, $\left.180^{\circ}, 240^{\circ}, 300^{\circ}\right)$. The upright orientation was defined as fingers/toes pointing upwards $\left(0^{\circ}\right)$.

\section{Procedure}

Participants sat in front of a computer screen, positioned $60 \mathrm{~cm}$ distant from the participant's eyes. Stimuli presentation was controlled with E-Prime (Psychology Software Tools Inc., Pittsburgh, USA). After the presentation of each stimulus, participants verbally judged the laterality (left or right) of hands or feet, as quickly and accurately as possible. The stimulus remained visible on the screen until the response was given. RTs were automatically recorded by a microphone connected to the computer. Response accuracy was manually recorded by an experimenter. Participants were tested in one experimental session consisting of four blocks. Two blocks contained 96 pictures of hands and the other two blocks contained 96 pictures of feet. During each of the two blocks of hands and feet stimuli, participants adopted a specific upper limb posture, as depicted in Fig. 1: in the "right hand in front" condition, participants positioned their right hand on the side of their right knee with their right thumb up, they were also asked to rotate their left arm behind their back in order to touch their back with the left palm. In the "right hand in back" condition they held their right hand behind their back and their left hand in front. The same postural manipulation was used for the blocks of feet stimuli. The hand positioned beside the knee, was not hidden from view, but participants were asked to fixate on the centre of the screen in front of them and their gaze was monitored online by the experimenter. The order of conditions and presented stimuli were counterbalanced across participants. After each block, participants completed a questionnaire in which they were asked to rate the 
Fig. 1 Experimental setting. Participants sat in front of the computer screen. A microphone on the desk recorded the verbal response time during task execution. In the "right hand in front" condition participants held the right hand on the side of the right knee and the left hand behind the back (a), vice versa for the "right hand in back" condition (b)
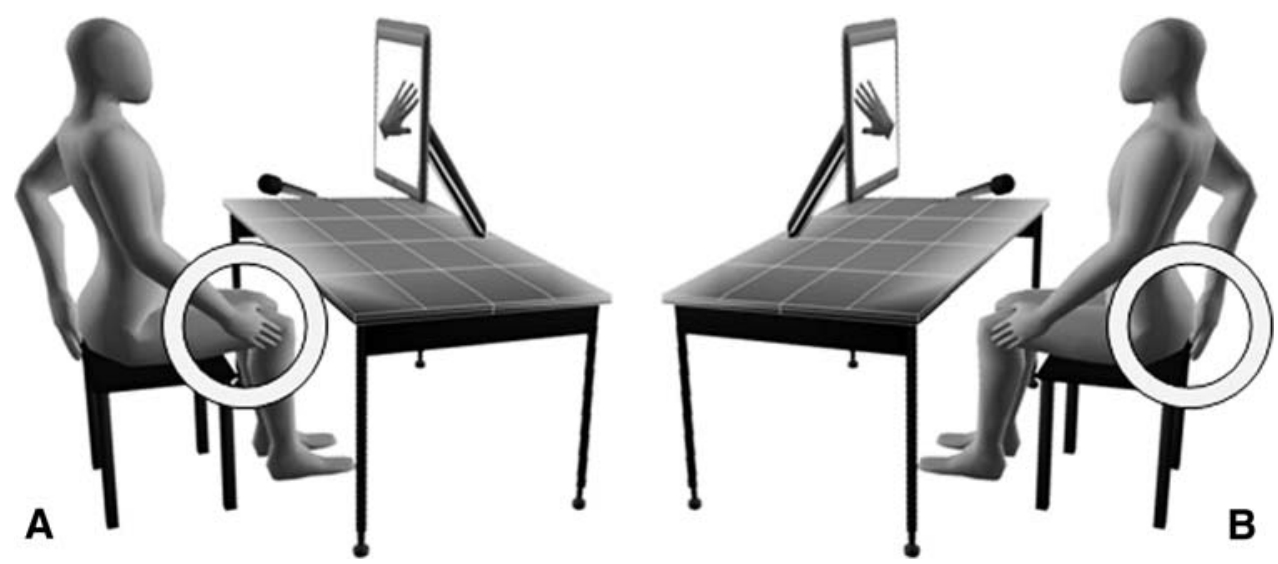

difficulty they had in judging the left and the right stimuli in the postural condition they had just experienced. These personal difficulty ratings were collected using a visual analogue scale (VAS), where participants were asked to mark a $10 \mathrm{~cm}$ long line representing their rating: values equal to zero meant "no difficult at all", values equal to 10 meant "absolutely impossible".

\section{Data analysis}

Previous studies investigating motor imagery and focusing on behavioural performance (Cooper and Shepard 1975; Sekiyama 1982; Parsons 1987, 1994; Wohlschlager and Wohlschlager 1998), or on physiological responses with positron-emission tomography (Bonda et al. 1995), and functional magnetic resonance imaging (de Lange et al. 2006), showed that the effects of stimulus orientation and stimulus view (in a mental rotation task involving body parts) particularly affect RTs. Therefore, we decided to focus our analysis on RTs only. RT was defined as the time between stimulus onset and the participant's verbal response. Previous studies using similar laterality tasks reported that RTs to hand stimuli range between 500 and 3,500 ms. Therefore, trials in which RTs were faster than $500 \mathrm{~ms}$ or slower than $3,500 \mathrm{~ms}$ and with allowable RTs but incorrect responses were excluded from the data analyses (total loss $8 \%$ of trials). In experiments 1 and 2, RTs were analyzed by means of a repeated measures analysis of variance (ANOVA), including stimulus (hands, feet), posture (right in front; right in back), laterality (left stimuli, right stimuli), orientation $\left(0^{\circ}, 60^{\circ}, 120^{\circ}, 180^{\circ}, 240^{\circ}, 300^{\circ}\right)$ and view (dorsum, little toe/finger, big toe/thumb, plantar/palm) as main factors. Post hoc comparison was performed using the NewmanKeuls test $(P<0.05)$. VAS scale representing personal ratings were analyzed by means of repeated-measure three-way ANOVAs with stimulus (hand, foot), posture (right hand in front, left hand in front) and laterality (left stimuli, right stimuli) as main factors.

\section{Results: experiment 1 (right-handed participants)}

Response time

Statistical analysis of RTs showed significant main effects of laterality $[F(1,13)=10.77 ; \quad P<0.006]$, orientation $[F(5,65)=15.68 ; \quad P<0.001], \quad$ view $\quad[F(3,39)=45.07$; $P<0.001]$ and the significant two-way interactions posture by laterality $[F(1,13)=14.11, P<0.002]$, stimulus by view $[F(3,39)=19.45 ; \quad P<0.001], \quad$ orientation by view $[F(15,195)=9.8 ; P<0.001]$, as well as three-way interactions stimulus by posture by laterality $[F(1,13)=10.53$; $P<0.006]$, stimulus by orientation by view $[F(15,195)=9.47 ; P<0.001]$, laterality by orientation by view $[F(15,195)=14.75 ; P<0.001]$ and the four-way interaction stimulus by laterality by orientation by view $[F(15,195)=3.95 ; P<0.001]$. The laterality main effect was accounted for by the faster performance for right stimuli $(1,206 \mathrm{~ms})$ with respect to the left stimuli $(1,257 \mathrm{~ms})$ $(P<0.001)$. The orientation main effect was accounted for by the slowest performance at $180^{\circ}(1,338 \mathrm{~ms})$ and at $120^{\circ}$ $(1,273 \mathrm{~ms})$ compared with all other orientations (respectively, $P<0.012$ and $P<0.001)$. The view main effect was accounted for by faster responses for stimuli seen from the dorsum view $(1,090 \mathrm{~ms})$ and the thumb view $(1,168 \mathrm{~ms})$ with respect to all the others views (all $P<0.0035$ ). The two posture effects will be described below.

\section{Posture effects}

The significant stimulus by posture by laterality interaction shows that differences between the two postural conditions varies for left versus right hands but not for left versus right feet showing, as expected, that posture effects are present for hands, but not for feet (Fig. 2).

Moreover, this interaction showed that this effect also depended on which hand was seen. Thus, RTs increased when judging right hands with the right hand behind the back (compared to right hand in front), but not when 
Fig. 2 Posture effect (righthanded participants). Data relative to pictures of hands and feet. The difference in terms of RTs related to the two hand postures varies between left versus right hands: slower responses for "right hand stimuli" when the right hand is behind the back. There were differences between left and right feet. Error bars represent standard error

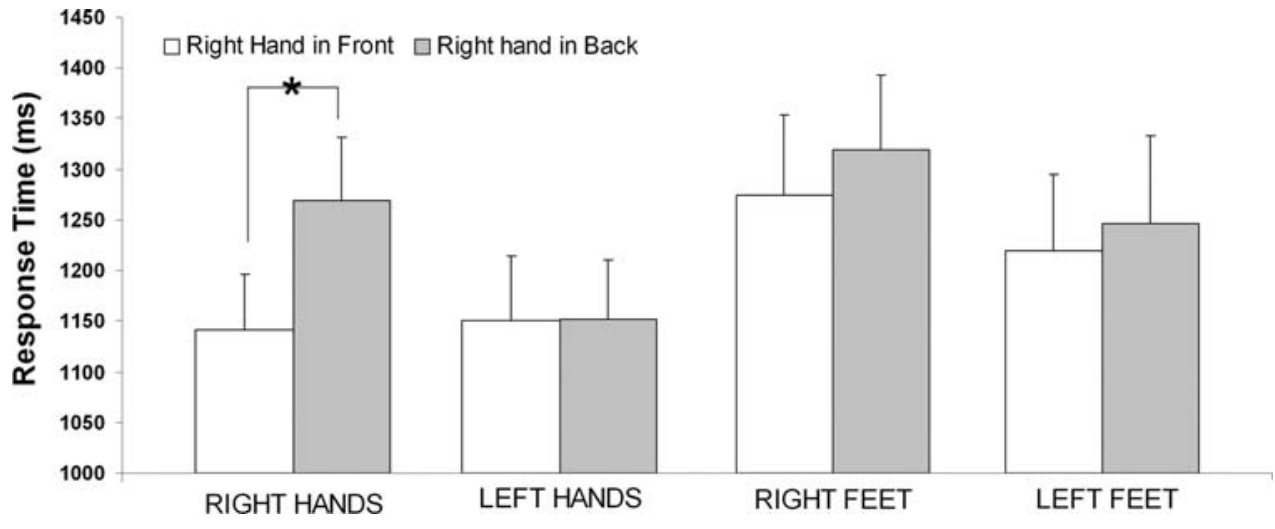

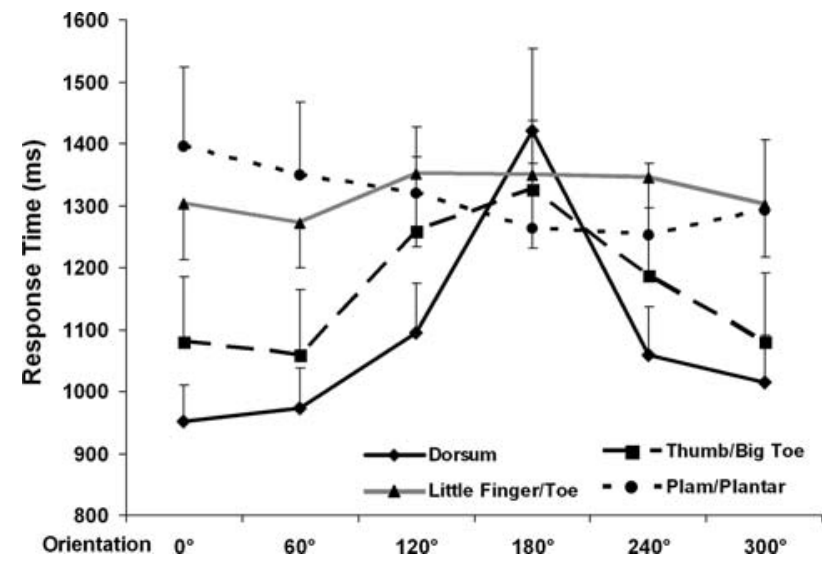

Fig. 3 View by orientation interaction effect (right-handed participants). The typical psychophysical profile of the mental rotation tasks is modulated by the view of the stimulus that has to be processed: the more difficult and unusual is the view, the more affected is the mental rotation, whit a progressive increase of the response time for usually easy orientations. Error bars represent standard error

judging left hands with the right hand in front (compared to right hand in back). These effects were not observed for feet judgments.

\section{Unfamiliar body-part images}

The significant orientation by view interaction shows that the often observed orientation effects in mental rotation of body parts are critically affected by the view of the body part. In particular, we note that the RT curve is non-mono- tonical at $180^{\circ}$ for stimuli seen in familiar views (dorsum; thumb/big toe), but flat for stimuli seen in unfamiliar views (little finger/toe, palm/plantar) and thus not depending on the angle of orientation (Fig. 3).

Thus, stimuli seen from the dorsum view at $180^{\circ}$ $(1,423 \mathrm{~ms})$ were the slowest compared with all other orientations in this view (all $P<0.001$ ). Stimuli seen from the thumb view at $180^{\circ}(1,320 \mathrm{~ms}), 120^{\circ}(1,268 \mathrm{~ms})$ and $240^{\circ}$ $(1,192 \mathrm{~ms})$ were not statistically different one another (all $P>0.32$ ), but they were significantly slower than images presented at $0^{\circ}(1,073 \mathrm{~ms}), 60^{\circ}(1,069 \mathrm{~ms})$ and $300^{\circ}$ $(1,086 \mathrm{~ms})$ (all $P<0.05)$. For stimuli presented from the little finger/toe view, there were no significant differences as a function of the stimuli orientation (all $P>0.42$ ). For stimuli presented from the palm/plantar view RTs were not statistically different across all orientations (all $P>0.17$ ) except for $0^{\circ}$ that were slower than $180^{\circ}(P<0.032)$ and at $240^{\circ}$ $(P<0.035)$ (Table 1; Figure 4).

General effect of stimulus view

Hands and feet seen from the dorsum view were processed faster than all the other views, but did not differ between them. Indeed, the statistically significant stimulus by view interaction indicated that RTs for hands $(1,105 \mathrm{~ms})$ and feet $(1,074 \mathrm{~ms})$ seen from the dorsum view were not statistically different $(P=0.53)$, but that they were processed faster than other views (all $P<0.0003$ ), except hands seen from the thumb view (Table 2).

Table 1 Familiarity with body parts

\begin{tabular}{lccllll}
\hline & $0^{\circ}$ & $60^{\circ}$ & $120^{\circ}$ & $180^{\circ}$ & $240^{\circ}$ & $300^{\circ}$ \\
\hline Dorsum & $954(57)$ & $976(64)$ & $1,099(79)$ & $1,423(133)$ & $1,064(78)$ & $1,021(76)$ \\
Thumb/big toe & $1,073(105)$ & $1,069(104)$ & $1,268(120)$ & $1,320(112)$ & $1,192(108)$ & $1,086(112)$ \\
Little finger/toe & $1,321(89)$ & $1,270(72)$ & $1,379(118)$ & $1,346(117)$ & $1,355(95)$ & $1,317(84)$ \\
Palm/plantar & $1,417(127)$ & $1,367(118)$ & $1,346(107)$ & $1,262(107)$ & $1,261(116)$ & $1,295(115)$ \\
\hline
\end{tabular}

Mean (standard error) of response times as a function of stimulus orientation and stimulus view 


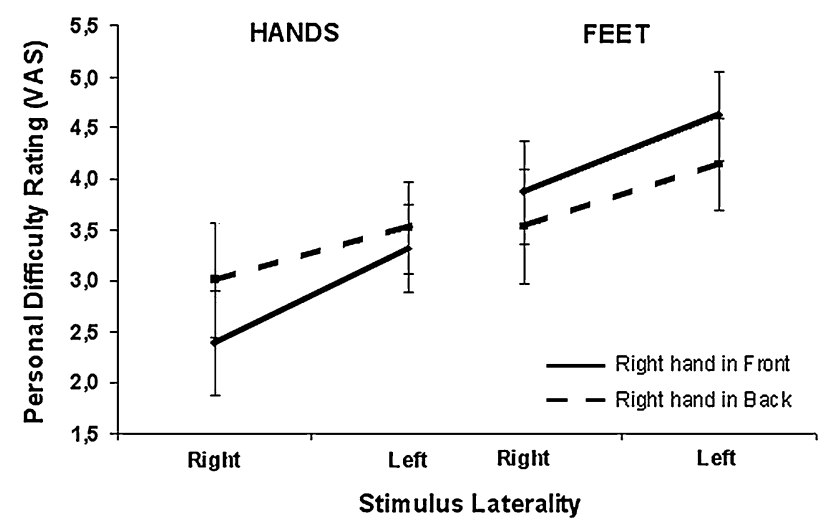

Fig. 4 Difficulty personal ratings (right-handed participants). Personal reports showed that rating the right hand with one's own right hand behind the back is more difficult than with the right hand in front. This was not true for the left hands. The difference between left and right feet was not related to postural variations. Error bars represent standard error

Table 2 Effect of view

\begin{tabular}{lllll}
\hline & Dorsum & Little finger/toe & Thumb/big toe & Palm/plantar \\
\hline Hands & $1,105(86)$ & $1,342(102)$ & $1,104(89)$ & $1,213(91)$ \\
Feet & $1,072(100)$ & $1,324(118)$ & $1,233(108)$ & $1,437(130)$ \\
\hline
\end{tabular}

Mean (standard error) of response times as a function of stimulus kind and stimulus view

\section{Differential view's effects on hands versus feet}

The significant stimulus by orientation by view interaction indicated that in the comparison between plantars (feet) versus palms (hands) and little toe (feet) versus little finger (hands), RTs for feet were generally slower than those for hands. This was true also in the comparison between images depicting the thumb versus big toe, but only when rotated at $240^{\circ}$. No differences in RTs were recorded between hands and feet both seen from the dorsum view.

\section{Stimuli's view and laterality effects}

The significant interaction laterality by orientation by view showed that no differences were found between left and right stimuli (hand and feet together) when pictures were seen from the dorsum view and rotated in all the orientations. When pictures were seen from the thumb/big toe view, RTs for left and right stimuli were different for rotations of $60^{\circ}, 120^{\circ}, 240^{\circ}$. For stimuli seen from the little finger/toe view, RTs for left and right stimuli were different for rotations of $120^{\circ}$ and $240^{\circ}$. This was also true for stimuli seen from the palm/plantar view and rotated of $300^{\circ}$.

\section{Difficulty ratings}

The effect of the posture manipulation was particularly evident for hands stimuli: the difference for difficulty ratings between the two postural conditions in the judgment of the corresponding stimulus was larger for hands than for feet. The ANOVA conducted on the difficulty ratings showed significant main effects for stimulus $[F(1,15)=4.8$; $P<0.04]$ and laterality $[F(1,15)=7.4 ; P<0.015]$. The stimulus main effect was accounted for by easier rating for hands (3.0) with respect to feet $(4.0)(P<0.04)$. The laterality main effect was accounted for by easier ratings for right stimuli (3.2) with respect to left ones $(3.9)(P<0.02)$. This effect, i.e. the preference for hands with respect to feet, judged to be more difficult, was noted previously (Ionta et al. 2007) and was not systematically analyzed here.

\section{Summary: experiment 1}

The data show a progressive disruption of the mental rotation function for stimuli representing body parts from unfamiliar views. The distribution of RTs along the typical psychophysical profile for mental rotation tasks is progressively flattened for stimuli shown from the palm/plantar and the little finger/toe views, compatible with a dependence of mental spatial transformations of body parts on anatomical constraints. A major result of experiment 1 is that when participants hold the right hand behind the back this leads to costs in response speed when judging the laterality of hand pictures. This posture effect was only found for pictures of right hands, and absent for the judgement of left hands and feet. In order to further characterise the mechanisms underlying this posture effect, we carried out experiment 2 using the same experimental setup and procedure, but now tested a group of ten left-handed participants.

\section{Results: experiment 2 (left-handed participants)}

\section{Response time}

Statistical analysis of RTs showed significant main effects of stimulus orientation $[F(5,40)=21.4 ; P<0.001]$ and view $[F(3,24)=21.8 ; P<0.001]$. Significant 2 -way interactions were found for laterality by view $[F(3,24)=7.2$; $P<0.0013]$ and orientation by view $[F(15,120)=22.1$; $P<0.001]$. Significant three-way interactions were found for posture by laterality by view $[F(3,24)=5.9 ; P<0.004]$ and for laterality by orientation by view $[F(15,120)=4.6$; $P<0.001]$. A significant four-way interaction was found for posture by laterality by orientation by view $[F(15,120)=3.4 ; P<0.001]$. The main effect of laterality was not significant $[F(1,8)=2.02 ; P=0.19]$. The orientation main effect was accounted for by the slowest performance at $180^{\circ}(1,193 \mathrm{~ms})$ compared with all other orientations (all $P<0.001)$ and by longer RTs for stimuli presented at $120^{\circ}$ 
Fig. 5 Absence of the posture effect (left-handed participants). Data relative to hands and feet. No difference in terms of RTs between the two postural conditions neither for the stimuli depicting left hands, right hands, left feet and rightfeet. Error bars represent standard error

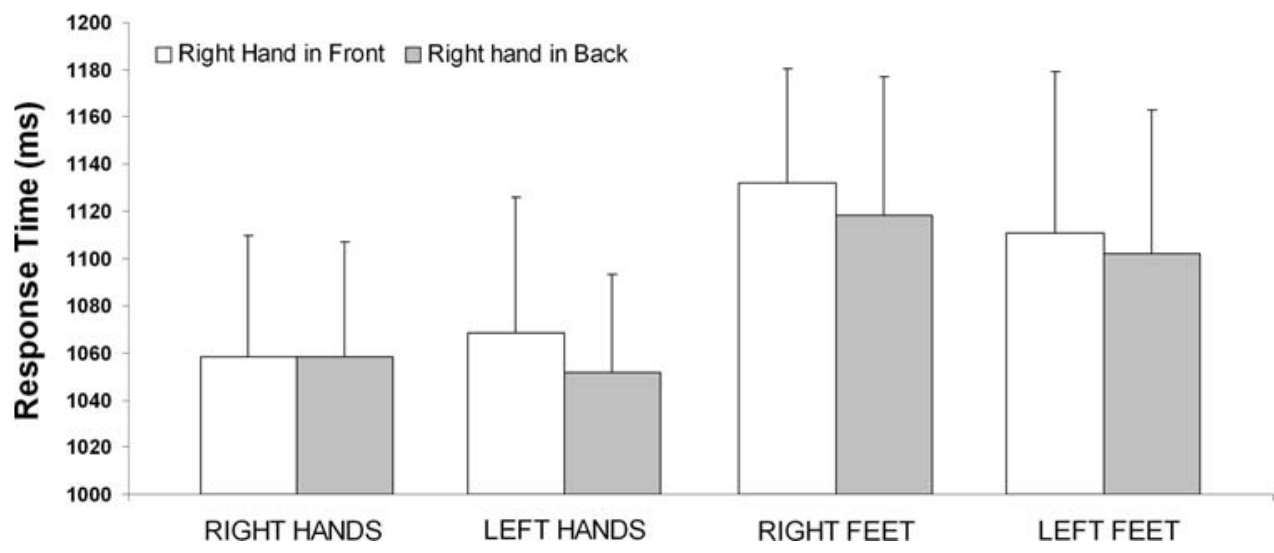

$(1,096 \mathrm{~ms})$ with respect to stimuli presented at $300^{\circ}$ $(1,078 \mathrm{~ms})(P<0.02)$. The view main effect was accounted for by the slowest performance obtained with stimuli presented from the little finger/toe view $(1,204 \mathrm{~ms})$ with respect to all the other views (all $P<0.001$ ). These effects are comparable to those of experiment 1 . The main differences between experiments 1 and 2 are the laterality main effect, found to be significant in experiment 1 but not in experiment 2 , and the three-way interaction between stimulus, posture and laterality, found to be significant in experiment 1 but not in experiment $2[F(1,8)=0.04 ; P=0.8]$ (Fig. 5). The detailed results are described below.

\section{Posture effects}

Although in left-handed participants pictures of left hands led to slowest RTs when the left hand was in the back (see Fig. 5) this was not found to be significant (no significant interaction stimulus by posture by laterality interaction; $F(1,8)=0.04 ; P=0.84)$. This indicates that there was no difference between the two postural conditions for left and right hands and feet. However, in contrast to experiment 1 , the significant interaction between posture, laterality and view $[F(3,24)=5.9 ; P<0.004]$ showed that for right stimuli seen from the dorsum view, participants were faster when keeping the right hand in front (995 ms) than when keeping the right hand behind the back (1,090 ms) $(P<0.03)$, while for stimuli seen from the palm/plantar view, participants were slower when keeping the right hand in front $(1,152 \mathrm{~ms})$ than keeping it behind the back $(1,097 \mathrm{~ms})(P<0.04)$. For left stimuli participants were faster with the right hand in front $(996 \mathrm{~ms})$ then behind the back $(1,084 \mathrm{~ms})$, only when stimuli were seen from the palm/plantar view $(P<0.04)$.

\section{Unfamiliar body-part images}

As in experiment 1, left-handed participants' RTs also showed a significant interaction between orientation and view $[F(15,120)=22.1 ; P<0.001]$ describing the disruption of the typical mental rotation profile (factor orientation) dependant on view of the stimuli as observed in righthanded participants (Fig. 6).

\section{General effect of stimulus view}

As in experiment 1 , hands and feet stimuli-seen from the little finger/toe view-were processed slower than all the other views in left-handed participants. Moreover, the statistically non-significant stimulus by view interaction indicated that RTs for hands and feet stimuli (compared for the same view) were similar.

\section{Differential view's effects on hands versus feet}

In contrast to the finding with right-handed participants, for left-handed participants, there was no significant stimulus by orientation by view interaction. This indicates that when comparing response speed as a function of the view, hands and feet were processed with the same speed relative to the same rotation angle.

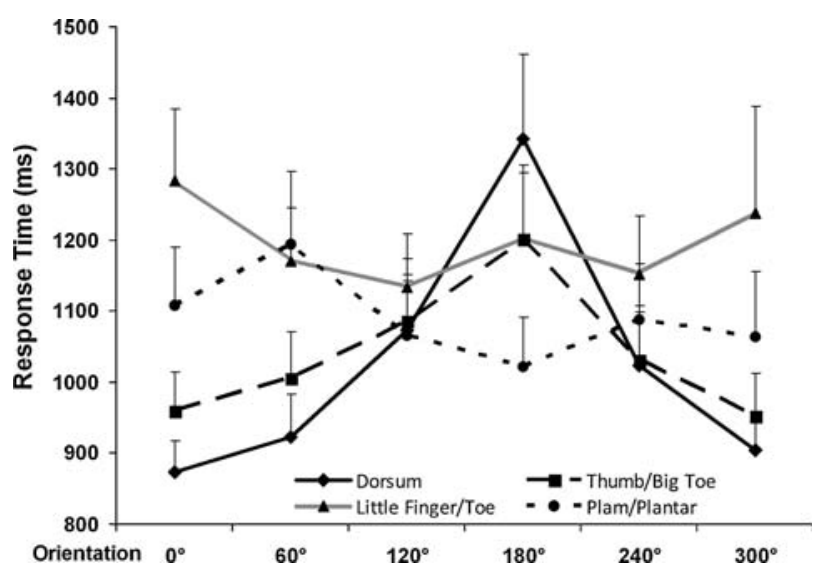

Fig. 6 View by orientation interaction effect (left-handed participants). RTs are distributed along the typical mental rotation functions for views which elicit biomechanically familiar movements (dorsum and thumb views). Error bars represent standard error 
Stimuli's view and laterality effects

As for right-handed participants, left-handed participants' performance also showed a significant interaction between laterality by orientation by view. This revealed that only for stimuli (hands and feet together) seen from the little finger/ toe view and rotated of $300^{\circ}$, left ones $(1,182 \mathrm{~ms})$ were slower than right ones $(951 \mathrm{~ms})(P<0.001)$.

\section{Difficulty ratings}

Compatible with RTs, there were no differences in difficulty ratings with respect to stimulus laterality for hands or for feet (posture and laterality main effects, as well as stimuli by laterality interaction, were not significant, all $P>0.6)$. The ANOVA for difficulty ratings showed a significant main effect of stimulus $[F(1,9)=11.54$; $P<0.008$ ] accounted for by easier ratings for hands (2.9) as compared to feet $(4.1)(P<0.008)$. This result was also confirmed by the significant three-way interaction between stimulus, posture and laterality $[F(1,9)=7.84$; $P<0.02$ ], showing that ratings for same stimuli judged in the two postural conditions were not statistically different (all $P>0.1$ ).

\section{Summary: experiment 2}

In left-handed participants there was no interference of arm posture on the mental rotation as found in right-handed participants, although they did show similar effects on response speed concerning stimulus view and orientation. In particular, we note that the typical profile for the mental rotation task is maintained for stimuli seen from the dorsum view, is still recognisable for the thumb/big toe view, but is almost absent for the palm/plantar view and absent for the little finger/toe view (Fig. 6).

\section{Experiment 3}

In order to further investigate the right hand posture effect found in experiment 1 and in order to investigate a potential contribution of the hand's visibility, we ran experiment 3 . Thus, it could be argued that what we have called here the posture effect may be due to the visibility of the (left) hand that was positioned on the knee, rather than to the position of the (invisible right) hand behind the back, leading to relative response facilitation for pictures showing left hands with respect to right hands ones. This was controlled in experiment 3 and the hands were not visible in either of the condition in right-handed participants.

\section{Methods}

Participants

Eight healthy participants (2 females) aged 25-34 years $(M=28.8$ years, $\mathrm{SD}=2.9)$ were enrolled in the experiment. All participants were right-handed according to a standard handedness inventory (Briggs and Nebes 1975). The average handedness score of this group was 4.3.

Procedure

Procedure was the same as in experiments 1 and 2, but the subjects' hand in front was hidden under the table. Since the main question of experiment 3 concerned any potential effect of hand visibility on the judgement of hands, we did not include the feet stimuli.

Data analysis

Experiment 3 included only hand judgments. Therefore, data analysis did not include the "stimulus" factor. RTs were analyzed by means of a repeated measures ANOVA, including posture (right in front; right in back), laterality (left stimuli, right stimuli), orientation $\left(0^{\circ}, 60^{\circ}, 120^{\circ}, 180^{\circ}\right.$, $240^{\circ}, 300^{\circ}$ ) and view (dorsum, little finger, thumb, palm) as main factors. Post hoc comparison was performed using the Newman-Keuls test $(P<0.05)$.

\section{Results: experiment 3}

\section{Response time}

Statistical analysis of RTs showed the significant main effects of stimulus orientation $[F(5,30)=12.8 ; P<0.001]$ and view $[F(3,18)=37.8 ; P<0.001]$. Significant two-way interactions were found for posture by laterality $[F(1,6)=8.7 ; \quad P<0.025]$ and for orientation by view $[F(15,90)=4.2 ; P<0.001]$. A significant three-way interaction was found for posture by laterality by orientation $[F(5,30)=7.1 ; P<0.001]$. A four-way significant interaction was found for posture by laterality by orientation by view $[F(15,90)=6.1 ; P<0.001]$. The orientation main effect was accounted for by the slowest performance for stimuli presented at $180^{\circ}(1,391 \mathrm{~ms})$ with respect to all the other orientations (all $P<0.003$ ) and by longer RTs for stimuli presented at $120^{\circ}(1,247 \mathrm{~ms})$ and at $240^{\circ}(1,268 \mathrm{~ms})$ with respect to stimuli presented at $60^{\circ}(1,166 \mathrm{~ms})$ and $300^{\circ}$ $(1,122 \mathrm{~ms})$ (all $P<0.03)$. In this way, the typical profile of RTs for mental rotation is replicated as well as in experiments 1 and 2. The view main effect was accounted for by the slowest performance obtained with stimuli presented 
from little finger $(1,335 \mathrm{~ms})$ and palm $(1,343 \mathrm{~ms})$ with respect to dorsum $(1,120 \mathrm{~ms})$ and thumb $(1,092 \mathrm{~ms})$ views (all $P<0.001$ ). This view effect is comparable with that found in experiments 1 and 2 .

Posture effects

The posture effect of experiment 1 was confirmed in experiment 3 . This was reflected by a significant posture by laterality interaction, indicating the slowest RTs for right hands when the subjects' right hand was behind the back with respect to the three remaining conditions (all $P<0.02$ ). In particular, compared with right hand in front $(1,185 \mathrm{~ms})$, RTs increased while judging right hands with the right hand behind the back $(1,302 \mathrm{~ms})(P<0.04)$. RTs did not differ while judging left hands with the right hand in front $(1,221 \mathrm{~ms})$ or in back $(1,182 \mathrm{~ms})(P=0.57)$. Thus, in-line with experiment 1 , the posture effect was present for the judgment of right hands but not for left hands (Fig. 7). The three-way interaction between posture, laterality and orientation was accounted for by the slowest response for left stimuli at $180^{\circ}$ with the right hand in front $(1,504 \mathrm{~ms})$ and for right stimuli at $180^{\circ}$ and $240^{\circ}$ with the right hand in back (1,464 and 1,461 ms, respectively) with respect to all the other conditions (all $P<0.04$ ).

\section{Unfamiliar body-part images}

The significant orientation by view interaction replicates the results found in experiments 1 and 2, indicating a progressive disruption of the RTs profile for mental rotation of body parts as a function of the stimulus view. In particular the mental rotation profile is maintained for stimuli seen from the dorsum and the thumb views, but it is impaired for stimuli seen from the little finger and palm views (Fig. 8).

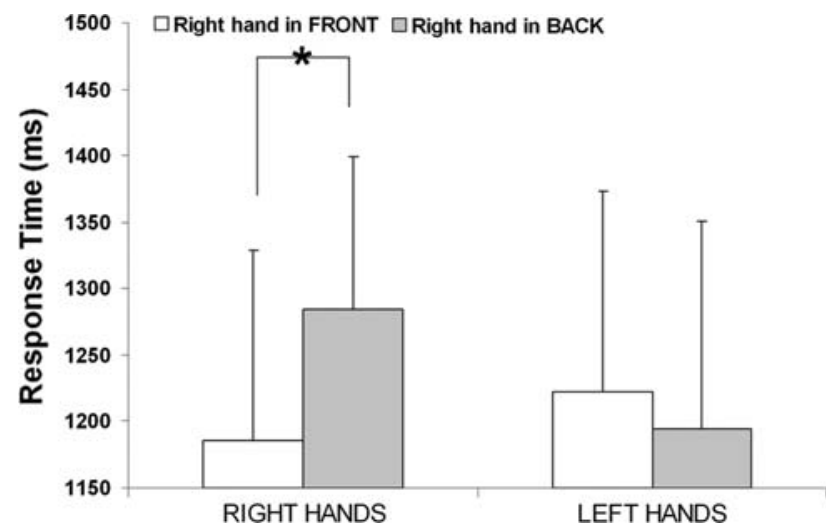

Fig. 7 Posture effect (control experiment). Data relative to pictures of hands. Difference in terms of RTs between the right and left hands as a function of the postural conditions: RTs "right stimuli" are slower when the right hand is behind the back, but RTs for "left stimuli" do not differ between the two postures. Error bars represent standard error

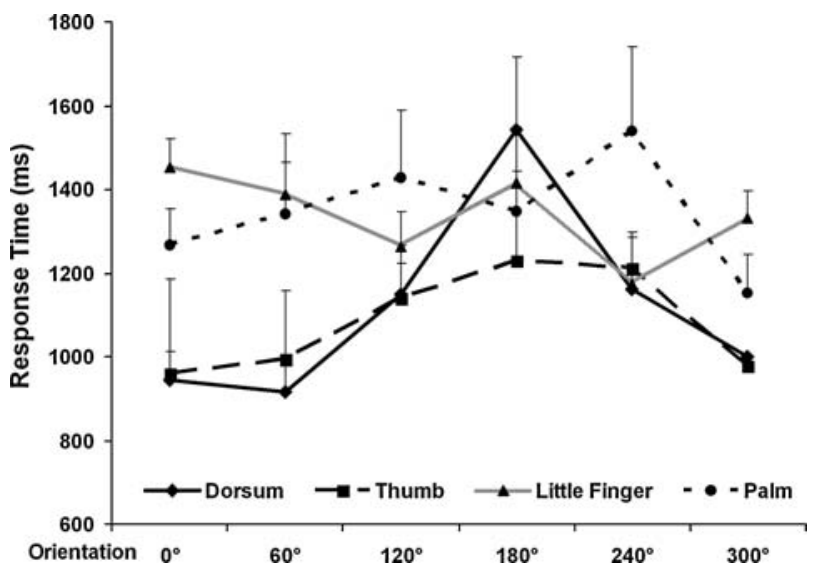

Fig. 8 View by orientation interaction effect (control experiment). Progressive disruption of the RTs profile as a function of the stimulus view

\section{Summary: experiment 3}

Presence (experiment 1) or absence (experiment 3 ) of hand visibility during task performance leads to a comparable posture effect. Thus, when participants hold the right hand behind the back this leads to costs in response speed when judging the laterality of hand pictures independently of whether the hand can be seen in the peripheral visual field or not. In addition, non-visibility of the hand does not modify the progressive flattening of the RTs typical for the mental rotation profile that we observed as a function of the stimulus view (as found in experiments 1 and 2).

\section{General discussion}

The present study explored whether the effects of body posture on the speed of mental rotation of body parts are selective for the side or the body part for which the posture was modified. In three experiments participants were asked to perform verbal laterality judgments for pictures of hands and feet in two different upper extremity postures. Our data show that a distant and posterior arm posture (behind the back and distant with respect to the visual stimulus) as opposed to a proximal and anterior arm posture (on the knee) leads to RT costs, for images representing hand positions of the corresponding body side. This was found in right-handed (experiment 1), but not in left-handed participants (experiment 2) and did not depend on the visibility of the upper extremity (experiment 3 ).

\section{Posture}

Recent studies have shown that neurological alterations of body representations may bring about performance alterations 
in tasks requiring hand laterality judgements (Fiorio et al. 2006; Schwoebel et al. 2001). Patients with focal hand dystonia, affecting the right dominant hand, are slower than controls in mentally rotating pictures of a hand (corresponding to their affected body part) but not pictures of feet (Fiorio et al. 2006). Patients suffering from chronic arm pain also show slower RTs for mental rotation of the affected than the unaffected limb (Schwoebel et al. 2001). These and other observations are compatible with the ideas that the central body representation might be influenced by peripheral factors such as changes in pain and posture changes and that multisensory and sensorimotor mechanisms are important in body part representation and imagery (Arzy et al. 2006; Funk and Brugger 2008).

In experiments 1 and 3 postural effects on mental rotation were only present for the dominant right hand. This was corroborated by difficulty ratings showing a significant increase of perceived difficulty between the two conditions only in right-handed participants and only for the judgment of right hands. Experiment 3 replicated the results of experiment 1 , although the hand in front was not visible to subjects, showing a postural effect only for the right dominant hand. The data from all the experiments suggest that a body-part specific influence of proprioceptive information on mental rotation of body parts is present in right-handed people. These observed differences may also be related to other mechanisms such as those related to the participant's hand dominance or to mechanisms related to greater visual familiarity with different hand postures for right hands in general as there are many more right-handed people in the population (who are likely to move their dominant hand more often). If hand dominance would be a crucial mechanism, left-handed participants should show the opposite effect compared with right-handed participants: RTs should be modulated as a function of the posture of the left hand, but only for stimuli depicting left hands and not for right hands (and not for feet). If visual familiarity were to play a role, RTs should be modulated as a function of the depicted hand showing facilitation for right hands. Finally, both mechanisms as well as handedness-related mechanisms may be present. In order to disentangle between these different mechanisms we carried out experiment 2 in lefthanded participants. Our results in this population showed that the time required for judging the laterality of hands (or feet) was not influenced by arm posture. This was corroborated by the absence of difference in the difficulty ratings about the two postural conditions. Thus, body-part specific posture effects on mental rotation were found only in righthanded participants.

What might be the reason for the presence/absence of the posture effect? It is not clear yet whether the difference between left and right hand stimuli found in experiment 1 and 3 , but not in experiment 2 , is due to the more frequent use of the own right hand (in right handers when compared with the left hand in left handers) leading to a greater lateralization for right handers when compared with left handers (Gentilucci et al. 1998). Because our left-handed participants did not show the posture effect neither for the left hand nor for the right hand, we suggest that not only handedness but also other factors, such as visual familiarity, may be involved. Visual familiarity may counteract mechanisms related to manual dominance in left-handed participants, whereas both mechanisms (handedness; visual familiarity) may summate in right-handed participants. Thus, for right-handed participants, the effects of handedness and visual familiarity are congruent (greater use of right hand, greater familiarity for right hands of others), while for left-handed participants, handedness and visual familiarity are incongruent (greater use of left hand, greater familiarity for right hands of others). An alternative mechanism is that proprioceptive representations may differ for left and right hands in right handers (causing the observed mental rotation differences), but not in left handers. In this case, the lack of posture effects for stimuli depicting left hands in right-handed people could be explained by the fact that in these participants, the mental spatial transformation of the right hand is based on more proprioceptive dependant processes than those involved for the left hand. Moreover, left-handed people may not have the posture effect because they use their non-dominant hand more often and more expertly than right-handed people, because, for example many tools are designed for right-handed people (scissors, peelers, openers, video cameras, etc.) leading to greater bimanuality or ambidextrousness in left handers. The decreased left-right asymmetry with respect to handedness in keyboard players (Amunts et al. 1997) would also be compatible with this and suggests with respect to the present data that left-handed people may have developed fine-tuned motor and proprioceptive representations of both hands. This last point is compatible with less extreme handedness scores in our left-handed participants (if compared to the right-handed participants) and the observation that right handers processed right stimuli (hands and feet) faster than left stimuli. Faster RTs for mental rotation of right body parts with respect to left body parts has been reported by several studies involving right-handed healthy participants (Parsons 1987; Gentilucci et al. 1998; Ionta et al. 2007; Funk and Brugger 2008), upper limb amputees (Nico et al. 2004) and congenital amputees (Brugger et al. 2000), suggesting that right handers are generally faster with right body parts. Our data support these previous findings and also extend them by showing that left-handed participants did not show either laterality preference and indicating that mental rotation of body parts depends at least on motor (and/or proprioceptive) and visual mechanisms that seem to interact differently in right- and left-handed people. 


\section{Orientation}

In seminal work on mental rotation, it was shown that RTs and response accuracy are distributed on a psychophysical profile, so that the characteristics of real spatial transformations would paste in mental spatial transformations (Shepard and Metzler 1971; Parsons 1994). Thus, people tend to compare the stimulus they see with a representation of it seen in its canonical orientation that is upright. In our case the upright orientation was defined as fingers/toes pointing upwards $\left(0^{\circ}\right)$ and RTs were distributed along the typical psychophysical profile for mental rotation tasks, following a non-monotonicity at $180^{\circ}$. The results in right- and lefthanded people (experiments 1 and 2) are in-line with the pre-existent studies demonstrating mental rotation functions for hands and feet judgments. However, it is important to emphasize that in localising body parts, proprioceptive information also needs to be integrated with other modalities in order to obtain a complete and continuously updated representation of the body.

\section{View}

The mental rotation function was affected by the factor "view" of the bodily stimuli in right- and left-handed people. This effect is in-line with the previous studies showing that RTs are longer for the mental rotation of stimuli corresponding to body part positions that are difficult to maintain or unfamiliar (Petit et al. 2003), and with literature showing that RTs are sensitive to the view from where the actual body part of the subject is seen during mental rotation (Parsons 1994). Thus, especially palm/plantar views seem to be slower than dorsum views (Ionta et al. 2007). In the present study, from the direct comparison between hands and feet as a function of the view, it is possible to conclude that the little finger view (for the hands) and the plantar view (for the feet) are the most biomechanically difficult mental spatial transformations, probably because participants tended to imagine the rotation of their own hand and foot into the stimulus position.

\section{Orientation and view}

Previous studies showed that the mental rotation of body parts is sensitive to the orientation (Parsons 1987, 1994; Arzy et al. 2006; Overney and Blanke 2008) and the view (Parsons 1994; Petit et al. 2003; Ionta et al. 2007) of the stimuli. Yet, the present results indicate that there is interdependence between orientation and view showing that uncommon views are less sensitive to orientation changes. For example in all three present experiments, the mental rotation function (orientation effect) is preserved for hands seen from the dorsum view but not for stimuli seen from the palm view, suggesting that the interdependence (typical distribution of RTs along the psychophysical profile for mental rotation, disrupted as a function of the stimulus view) is present in right- and left handers and is independent from hand visibility. Even if "view" has been considered an important factor in laterality judgements of hand pictures (Parsons 1994), to our knowledge this is the first time that different views have been directly compared. The observation of biologically impossible and possible movements activates different neural structures (Stevens et al. 2000). Priming effects do not seem to influence (or are less influential for) tasks employing impossible postures (Daems and Verfaillie 1999). Finally, anatomical joint constraints seem to play an important role in the simulation of an action, resulting in different RTs for the imagery of anatomically possible and impossible movements (Parsons 1987; Petit et al. 2003). We suggest that the progressive disruption of the typical mental rotation function with respect to the stimulus view, regardless of hand dominance, can be seen as behavioural evidence for increases in biomechanical difficulty when simulating the rotation of one's own hand around the wrist. Notably, if participants would actually rotate their own hands in this way starting from the dorsum view, then the second view would be the thumb, the third would be the palm and the fourth would be the little finger. The same order was observed in our data: stimuli shown form the dorsum view determine the typical mental rotation function, followed by the thumb, palm and little finger, resulting in longer RTs for the longer and biomechanically more difficult rotation. This suggests a very close overlap in terms of shared properties between real and simulated actions.

\section{Concluding remarks}

By testing the influence of the handedness of the observer and through the manipulation of the arm posture of the observer we show that the mental rotation of body parts depends on motor and proprioceptive mechanisms. Since the posture effect differed between right- and left-handed participants our data suggest that additional visual mechanisms are important and that these different mechanisms modulate performance differently in both subject groups.

Acknowledgments For his invaluable suggestions, the authors would like to thank Prof. Gian Luca Romani. We would also like to thank the two anonymous reviewers for their helpful comments.

\section{References}

Amunts K, Schlaug G, Jancke L, Steinmetz H, Schleicher A, Dabringhaus A, Zilles K (1997) Motor cortex and hand motor skills: structural compliance in the human brain. Hum Brain Mapp 5:206-215 
Arzy S, Overney LS, Landis T, Blanke O (2006) Neural mechanisms of embodiment: asomatognosia due to premotor cortex damage. Arch Neurol 63:1022-1025

Bonda E, Petrides M, Frey S, Evans A (1995) Neural correlates of mental transformation of the body-in-space. Proc Natl Acad Sci USA 92:11180-11184

Briggs CG, Nebes RD (1975) Patterns of hand preference in a student population. Cortex 11:230-238

Brugger P, Kollias SS, Muri RM, Crelier G, Hepp_Reymond MC, Regard M (2000) Beyond remembering: phantom sensations of congenitally absent limbs. Proc Natl Acad Sci USA 97(11):61676172

Cooper LA, Shepard RN (1975) Mental transformations in the identification of left and right hands. J Exp Psychol Hum Percept Perform 104(1):48-56

Daems A, Verfaillie K (1999) Viewpoint-dependent priming effects in the perception of human actions and body postures. Vis Cogn 6(6):665-693

de Lange FP, Helmich RC, Toni I (2006) Posture influences motor imagery: an fMRI study. Neuroimage 33(2):609-617

Decety J, Jeannerod M, Prablanc C (1989) The timing of mentally represented actions. Behav Brain Res 34:35-42

Decety J, Jeannerod M, Germain M, Pastene J (1991) Vegetative response during imagined movement is proportional to mental effort. Behav Brain Res 42:1-5

Fadiga L, Buccino G, Craighero L, Fogassi L, Gallese V, Pavesi G (1995) Corticospinal excitability is specifically modulated by motor imagery: a magnetic stimulation study. Neuropsychologia 37:147-158

Fiorio M, Tinazzi M, Aglioti SM (2006) Selective impairment of hand mental rotation in patients with focal hand dystonia. Brain 129(Pt 1):47-54

Funk M, Brugger P (2008) Mental rotation of congenitally absent hands. J Int Neuropsychol Soc 14(1):81-89

Ganis G, Keenan JP, Kosslyn SM, Pascual-Leone A (2000) Transcranial magnetic stimulation of primary motor cortex affects mental rotation. Cereb Cortex 10:175-180

Gentilucci M, Daprati E, Gangitano M (1998) Right-handed and lefthanded have different representation of their own hand. Brain Res Cogn Brain Res 6(3):185-192

Gerardin E, Sirigu A, Lehericy S, Poline JB, Gaymard B, Marsault C, Agid Y, Le Bihan D (2000) Partially overlapping neural networks for real and imagined hand movements. Cereb Cortex 10(11):1093-1104
Grezes J, Decety J (2001) Functional anatomy of execution, mental simulation, observation, and verb generation of actions: a metaanalysis. Human Brain Mapp 12(1):1-19

Ionta S, Fourkas A, Fiorio M, Aglioti SM (2007) The influence of hands posture on mental rotation of hands and feet. Exp Brain Res 183:1-7

Kosslyn SM, Di Girolamo GJ, Thompson WL, Alpert NM (1998) Mental rotation of objects versus hands: neural mechanisms revealed by positron emission tomography. Psychophysiology 35(2):151-161

Kosslyn SM, Ganis G, Thompson WL (2001) Neural foundations of imagery. Nat Rev Neurosci 2(9):635-642

Nico D, Daprati E, Rigal F, Parsons L, Sirigu A (2004) Left and right hand recognition in upper limb amputees. Brain 127:120-132

Overney LS, Blanke O (2008) Impaired imagery for upper limbs. Brain Topogr (in press). doi:10.1007/s10548-008-0065-2)

Parsons LM (1987) Imagined spatial transformation of one's hands and feet. Cogn Psychol 19:178-241

Parsons LM (1994) Temporal and kinematic properties of motor behavior reflected in mentally simulated action. J Exp Psychol Hum Percept Perform 20(4):709-730

Parsons LM, Fox PT, Downs JH, Glass T, Hirsch TB, Martin CC, Jerabek PA, Lancaster JL (1995) Use of implicit motor imagery for visual shape discrimination as revealed by PET. Nature 375(6526):54-58

Petit LS, Pegna AJ, Mayer E, Hauert CA (2003) Representation of anatomical constraints in motor imagery: mental rotation of a body segment. Brain Cogn 51(1):95-101

Schwoebel J, Friedman R, Duda N, Coslett HB (2001) Pain and the body schema: evidence for peripheral effects on mental representations of movement. Brain 124:2098-2104

Sekiyama K (1982) Kinesthetic aspects of mental representations in the identification of left and right hands. Percept Psychophys 32(2):89-95

Shepard NR, Metzler J (1971) Mental rotation of three-dimensional objects. Science 171:701-703

Sirigu A, Duhamel JR, Cohen L, Pillon B, Dubois B, Agid Y (1996) The mental representation of hand movements after parietal cortex damage. Science 273:1564-1568

Stevens JA, Fonlupt P, Shiffrar M, Decety J (2000) New aspects of motion perception: selective neural encoding of apparent human movements. Neuroreport 11:109-115

Wohlschlager A, Wohlschlager A (1998) Mental and manual rotation. J Exp Psychol Hum Percept Perform 24(2):397-412 